\title{
BNM MS. 19 (OLIM A 16): MIRABILIA ORIENTALIA INTRODUCCIÓN, EDICIÓN Y TRADUCCIÓN*
}

\author{
Álvaro IBÁÑEz CHACóN \\ alvaroic@ugr.es \\ Universidad de Granada
}

\section{INTRODUCCIÓN}

El manuscrito 19 (olim A 16) de la Biblioteca Nacional de Madrid, del que ya Juan Iriarte reprodujo algunos fragmentos a mediados el siglo XVIII ${ }^{1}$, es un interesantísimo ejemplar de lo que se conoce como libro de computus, es decir, una suerte de manuscrito misceláneo con obras relacionadas con la computística medieval: crónicas, astronomía, pesos y medidas, medicina, alquimia, etc. ${ }^{2}$ Ciertamente, el manuscrito contiene ${ }^{3}$ : varios tratados de Beda, los Aratea de Germánico con escolios, extractos de Solino, Isidoro de Sevilla y Agustín de Hipona, algunas recetas alquímicas y la carta objeto de la presente edición, estando algunos tratados iluminados con magníficas miniaturas ${ }^{4}$.

\footnotetext{
* Trabajo realizado en el marco del Proyecto de Investigación $\mathrm{I}+\mathrm{D}+\mathrm{i}$ «Estudios sobre la transmisión y recepción de Paléfato y la exégesis racionalista de los mitos» (Ref.: FFI201452203-P), financiado por el MINECO.

${ }^{1}$ Juan Iriarte, Regiae Bibliothecae Matritensis, Codices Graeci Mss., vol. I, Madrid, 1769, pp. 203-205.

${ }^{2}$ Así de acuerdo con el más reciente estudio de Alejandro García Avilés, El tiempo y los astros. Arte, ciencia y religión en la Alta Edad Media, Murcia, Universidad de Murcia, 2001, pp. 47-52.

${ }^{3}$ Detalladas descripciones del contenido en Gustav Loewe y Wilhelm von Hartel, Bibliotheca patrum latinorum Hispaniensis, vol. I, Wien, Akademie der Wissenschaften, 1887, pp. 315-317; Inventario general de manuscritos de la Biblioteca Nacional, vol. I, Madrid, Ministerio de Educación Nacional, 1953, pp. 20-23; José M. ${ }^{a}$ Millás Vallicrosa, «El ms. 19 de la Biblioteca Nacional de Madrid y sus influencias clásica y oriental en la transmisión de las ciencias», Revista de Archivos, Bibliotecas y Museos, 67 (1959), pp. 119-126.

${ }^{4}$ Gracias a estas obras singulares el manuscrito ha sido bien estudiado, entre otros, por Paul von Winterfeld, «De Germanici codicibus», en AA.VV., Festschrift Johannes Vahlen zum Siebenzigsten Geburstag, Berlin, G. Reimer, 1900, pp. 393-407 (esp. pp. 395-396); John M. Burnam, «Recipes from Codex Matritensis A 16», University of Cincinnati Studies, 8/1 (1912), pp. 5-47, y «A Group of Spanish Manuscripts», Bulletin Hispanique, 22/4 (1920), pp. 229-233; Jesús Domínguez Bordona, Manuscritos con pinturas, Madrid, Centro de Estudios Históricos, vol. I, 1933, n. ${ }^{\circ}$ 411; Alfred Cordoliani, «Un manuscrit de comput ecclésiastique mal connu de la Bibliothèque Nationale de Madrid», Revista de Archivos, Bibliotecas y Museos, 57 (1951), pp. 5-35; Antonio dell’Era, «Una miscellanea astronomica medievale: gli Scholia Strozziana a Germanico», Atti della Accademia Nazionale dei Linzei. Memorie, 23/2 (1979), pp. $147-$ 265; Michael D. Reeve, «Some Astronomical Manuscripts», Classical Quarterly 30/2 (1980), pp. 508-522; Elisabeth S. Lott, The Florilegium of Cava 3, Madrid 19 and Paris 7418 [tesis doctoral inédita], Harvard University, 1980, y de la misma autora «The textual tradition of
} 
Se compone de 199 folios en pergamino de gran formato (395 x $270 \mathrm{~mm}$ ), aunque la foliación moderna, indicada en la esquina superior derecha, da 203 fols., pues hay un error de numeración y del fol. 104r se pasa al fol. 109r; añádanse dos folios en papel de guarda al principio y uno al final incorporados en la encuadernación del siglo XVIII; los fols. 43r, 44r-v y 114v están en blanco. Los cuadernillos son irregulares, generalmente cuaterniones, y solo se han conservado las signaturas de los cuadernos $14^{\circ}$ (fol. $103 \mathrm{r}$ ) y $15^{\circ}$ (fol. $111 \mathrm{r}$ ), consignadas en la esquina inferior derecha. El texto está copiado a dos columnas, sin intercolumnio y sin una impaginatio bien definida, con 47 líneas por columna, dejando amplios márgenes que, por lo general, no se han ocupado con las habituales anotaciones. Desde el punto de vista gráfico, presenta una escritura de transición hacia la gótica libraria ${ }^{5}$, conservando elementos de los sistemas escriturarios anteriores (sobre todo los signos abreviativos, cfr. infra), pero ya con la realización gráfica de particularidades góticas como la $a$ con el trazo vertical totalmente recto o la similitud entre $u$ y $n$, confundibles en secuencias del tipo un, um, in, mi, etc. ${ }^{6}$ La fecha de copia, por tanto, se sitúa en el siglo $\mathrm{XII}^{7}$, atendiendo a la existencia de un modelo en escritura beneventana ${ }^{8}$.

Así, pues, para ser rigurosos con la terminología empleada, el ms. 19 BNM no es simplemente un ejemplar «misceláneo» o un florilegium, pues, desde el punto de vista propiamente codicológico y paleográfico, Alfred Cordoliani detectó al menos 4 manos diferentes que comparten, sin embargo, una misma técnica escrituraria ${ }^{9}$, concluyendo que el manuscrito sería obra del trabajo conjunto de copistas

the Aratea of Germanicus Caesar: missing links in the $\mu$ branch», Revue d'histoire des textes, 11 (1983), pp. 147-158; Ann E. Knock, Wonders of the East: a synoptic edition of the Letter of Pharasmanes and the Old English and Old Picard translations (tesis doctoral inédita), London, Birbeck College, 1981; Alejandro García Avilés, «El manuscrito 19 de la Biblioteca Nacional de Madrid: un códice computístico de origen controvertido», Imafronte, 10 (1994), pp. 41-50.

${ }^{5}$ En un principio, Paul von Winterfeld, «De Germanici codicibus», art. cit., p. 395, seguido por Elias A. Loew, The Beneventan Script, Oxford-London, Clarendon Press-Humphrey Milford, 1914, p. 71, lo consideraron un ejemplar en escritura beneventana.

${ }^{6}$ Vid. Bernhard Bischoff, Latin Palaeography. Antiquity and the Middle Ages (trad. ing. D. Ó Croinin y D. Ganz), Cambridge, University Press, 1990, pp. 118 y sigs.; Giulio Batelli, Lezioni di paleografia, Città del Vaticano, Libreria Editrice Vaticana, 19994, pp. 202 y sigs.

${ }^{7}$ En general Inventario, cit., p. 23; Alfred Cordoliani, «Un manuscrit de comput...», art. cit., p. 6; Michael D. Reeve, «Some Astronomical Manuscripts», art. cit., p. 513; Elisabeth S. Lott, «The textual tradition of the Aratea...», art. cit., p. 151; Alejandro García Avilés, «El manuscrito $19 . . . »$, art. cit., p. 41; a principios del siglo lo adelantan Gustav Loewe y Wilhelm von Hartel, Bibliotheca patrum ..., op. cit., p. 315; José M. ${ }^{a}$ Millás Vallicrosa, «El ms. 19 de la Biblioteca...», art. cit., p. 119.

${ }^{8}$ Cfr. Alejandro García Avilés, «El manuscrito 19...», art. cit., p. 44; en cambio, John M. Burnam, «Recipes...», art. cit., p. 7 consideraba que el modelo podría ser un ejemplar italiano en semi-uncial del siglo VIII.

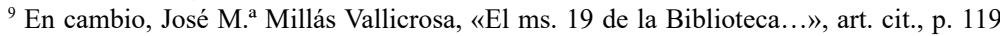
asevera que está escrito por una sola mano; el resto de estudios no se posiciona. 
de un mismo scriptorium $^{10}$; en este sentido, estaríamos ante un volumen unitario desde el punto de vista codicológico, pero pluritextual, en tanto que es de contenido diverso y está escrito por varias manos ${ }^{11}$.

En cuanto a la localización de ese scriptorium, en un principio, y sobre la base de discutibles «Spanish symptoms» ${ }^{12}$, se pensó que el ms. 19 había sido copiado en Santa María de Ripoll, pero muy pocos estudiosos aceptan hoy esta teoría, inclinados a considerarlo de origen italiano, en concreto procedente del sur de Italia o de la propia Sicilia ${ }^{13}$; así, los supuestos «caracteres hispanos» del manuscrito serían, en realidad, errores y vacilaciones ortográficas comunes al latín medieval ${ }^{14}$, no exclusivas de su desarrollo en la Península Ibérica ${ }^{15}$. Además, dado que el manuscrito es producto de varios manos diferentes, los «síntomas» podrían deberse a particularidades técnicas de uno $u$ otro amanuense $y$, por tanto, no determinarían el origen del conjunto, sino del copista en cuestión ${ }^{16}$. Además, los errores derivados de la incomprensión del antígrafo en beneventana son indicio para Anne Knock de que el manuscrito se copió en un área no beneventana ${ }^{17}$. En este sentido, no tenemos dudas de que el modelo se hallara copiado en este tipo de letra, tal y como demuestra un hecho significativo no señalado por los estudiosos precedentes: la presencia del típico signo beneventano usado a partir del siglo IX para marcar

${ }^{10}$ Alfred Cordoliani, «Un manuscrit de comput...», art. cit., p. 6. Este fenómeno de trabajo colectivo se atestigua también en scriptoria bizantinos; véase el minucioso estudio de Pasquale Orsini, «Pratiche collettive di scrittura a Bisanzio nei secoli IX e X», Segno e Testo, 3 (2005), pp. 265-342.

${ }^{11}$ Véase el ensayo de terminología propuesto por Marilena Maniaci, «Il codice greco "non unitario". Tipologie e terminologie», Segno e Testo, 2 (2004), pp. 75-107, matizado por Filippo Ronconi, I manoscritti greci miscellanei. Ricerche su esemplari dei secoli IX-XII, Spoleto, Centro Italiano di Studi sull'Alto Medioevo, 2007, pp. 1-32, ambos exclusivamente dedicados a códices griegos, pero sus propuestas son perfectamente aplicables a códices latinos.

${ }^{12}$ John M. Burnam, «Recipes...», art. cit., pp. 6-7 e Id., «A group...», art. cit., pp. 231-233, seguido por Alfred Cordoliani, «Un manuscrit de comput...», art. cit., p. 7; José M. a Millás Vallicrosa, «El ms. 19 de la Biblioteca...», art. cit., p. 126, y ya en su Assaig d'història de les idees fisiques i matematiques de la Catalunya medieval, Barcelona, Institució Patxot, 1931, pp. 237-240. Uno de los síntomas hispanos aducidos son los nombres de los vientos que aparecen en el diagrama del fol. 88r (no 87r), pero éstos son claramente añadidos posteriores, con una caligrafía típica del siglo XVIII, fecha de encuadernación del volumen.

${ }^{13}$ Entre otros Elias A. Loew, The Beneventan Script, op. cit., p. 71, Elisabeth S. Lott, «The textual tradition of the Aratea...», art. cit., p. 152, Alejandro García Avilés, «El manuscrito $19 . . . »$, art. cit., pp. 44 y sigs.

${ }^{14}$ Así lo han señalado, entre otros, Michael D. Reeve, «Some Astronomical Manuscripts», art. cit., p. 513, n. 23; Ann E. Knock, Wonders of the East..., op. cit., pp. 228-230.

${ }^{15}$ Véanse, en general, Karl P. Harrington, Medieval Latin, Chicago-London, The Chicago University Press, 19972; Pascale Bourgain y Marie-Clotilde Hubert, Le latin médiéval, Turnhout, Brepols, 2005; acerca del latín hispano-medieval vid. Juan Gil, «El latín tardío y medieval (siglos VI-VIII)», en R. Cano (ed.), Historia de la lengua española, Barcelona, Ariel, 2004, pp. 149-182.

${ }^{16}$ En este sentido, para justificar los supuestos síntomas hispanos, Alejandro García Avilés, «El manuscrito 19...», art. cit., p. 49 deja abierta la posibilidad de que el copista sea un hispano en un monasterio suritálico.

${ }^{17}$ Ann E. Knock, Wonders of the East..., op. cit., pp. 232-233. 
el valor interrogativo de los pronombres ${ }^{18}$, un ejemplo de lo cual se halla también en el texto que ahora editamos (cfr. infra).

Como hemos señalado anteriormente, el ms. 19 de la Biblioteca Nacional ha llamado la atención de numerosos investigadores desde el siglo XIX por la disparidad de obras que contiene, sobre todo por los tratados de contenido científico y astrológico, pero muy pocos estudios se centran en el interesante texto que aquí presentamos: la carta que supuestamente envió Farasmanes II al Emperador Adriano relatándole las maravillas de las regiones orientales ${ }^{19}$.

La epístola se ha conservado en dos redacciones sustancialmente diferentes: el testimonio más importante de la redacción a la que pertenece el ms. 19 es el Par. lat. NAL 1065, que presenta un texto menos epitomado y que conserva los elementos epistolares genui$\operatorname{nos}^{20}$; tales son los testimonios de la primera redacción: Parisinus NAL 1065, fols. 92v-95v $(=\mathbf{A})^{21}$, Casinensis 391 , fols. $82 \mathrm{v}-84 \mathrm{v}(=$ $\left.\mathbf{A}^{1}\right)^{22}$, Cauensis 3, fols. 397r-398r $\left(=\mathbf{A}^{2}\right)^{23}$, Matritensis 19, fols. 198r$199 \mathrm{r}\left(=\mathbf{A}^{3}\right)^{24}$ y Parisinus AFL 7418, fols. 268r-270v $\left(=\mathbf{A}^{4}\right)^{25}$

La otra redacción se caracteriza, principalmente, por haber eliminado los elementos epistolares y por haber sido interpolada con noticias procedentes de la leyenda de Alejandro $\mathrm{Magno}^{26}$, pero lo

${ }^{18}$ Cfr. Giulio Batelli, Lezioni di paleografia, op. cit., p. 127 y mucho más detallado Elias A. Loew, The Beneventan Script, op. cit., pp. 236-270.

${ }^{19}$ El estudio más completo sigue siendo la tesis inédita de Ann Knock ya citada, en la que se establecen las relaciones entre los testimonios, dividiendo en dos grandes grupos las recensiones latino-medievales; no obstante, sus hipótesis acerca del origen de la carta deben ser revisadas y reformuladas teniendo en cuenta el contexto histórico-cultural en el que fue compuesta y su carácter falsario. Las hipótesis de Knock son seguidas por Patrizia Lendinara en «De meraviglia in meraviglia», en Franco de Vivo (ed.), Circolazione di uomini, di idee e di testi nel Medioevo germanico. Atti del $25^{\circ}$ Convegno dell'Associazione italiana di filologia germanica, Cassino, Università di Cassino, 2002, pp. 177-229 y «The Letter of Fermes: Not only Marvels», en Kees Dekker, Karin Olsen y Tette Hofstra (eds.), The World of Travellers: Exploration and Imagination, Leuven, Peeters, 2009, pp. 31-60.

${ }^{20}$ Todos los miembros del mismo grupo presentan esta versión sesgada que, según Ann E. Knock, Wonders of the East..., op. cit., pp. 247-259 podría haber sido realizada por un cristiano en pos de la supresión de elementos paganos originarios.

${ }^{21}$ Preferimos emplear el sistema de siglas iniciado por Henry Omont, «Lettre à l'Empereur Adrien sur les merveilles de l'Asie», Bibliothèque de l'École des Chartes, 74 (1913), pp. 509515, continuado por Edmond Faral, «Une source latine de l'histoire d'Alexandre: la Lettre sur les merveilles de l'Inde», Romania, 43 (1914), pp. 199-215, 352-370 y Claude Lecouteux, De rebus in Oriente mirabilibus (Lettre de Farasmanes), Meisenheim am Glan, Verlag Anton Hain, 1979; Ann E. Knock, Wonders of the East..., op. cit., sin embargo, sigue un sistema diferente y más complejo que puede llevar a confusiones.

${ }^{22}$ Sigla MC en Ann E. Knock, Wonders of the East..., op. cit., pp. 221-223, que lo utiliza como base para su edición de esta recensión en pp. 933-940.

${ }^{23}$ Sigla $\mathbf{C}$ en Ann E. Knock, Wonders of the East..., op. cit., pp. 223-225 y $\mathbf{A}^{3}$ en Claude Lecouteux, «Deux nouveaux fragments de la Lettre à Adrien sur les merveilles de l'Asie», Romania, 104 (1983), pp. 113-124.

${ }^{24}$ Sigla $\mathbf{M}$ en Ann E. Knock, Wonders of the East..., op. cit., pp. 226-233.

${ }^{25}$ Sigla $\mathbf{P}$ en Ann E. Knock, Wonders of the East..., op. cit., pp. 233-234.

${ }^{26}$ Un estudio pormenorizado de la redacción II en Ann E. Knock, Wonders of the East..., op. cit., pp. 57 y sigs., y, con menos detalle, Lendinara, «De meraviglia», art. cit., pp. 177-186 $\mathrm{y}$ «The Letters of Fermes», art. cit., pp. 45-49; sobre el contexto histórico de su producción Asa 
más significativo es su circulación por ambientes culturales anglosajones, como prueba, entre otros detalles, la traducción al antiguo inglés en el Cotton Vitellius A.XV de la British Library de Londres (fols. $98 \mathrm{v}-106 \mathrm{v})^{27}$ y otra versión al antiguo francés en el ms. 14562 de la Bibliothèque Royale de Bruselas (fols. $5 \mathrm{v}-6 \mathrm{v}$ ) ${ }^{28}$. Los testimonios conservados de esta redacción son: $C o d$. $C I V 15$ de Estrasburgo $(=\mathbf{B})^{29}$ y Cotton Tib. B V de Londres, fols. $78 \mathrm{v}-86 \mathrm{v}(=\mathbf{D})^{30}$.

Finalmente, hay que tener en cuenta también la versión epitomada y alterada que presenta Gervasio de Tilbury en sus Otia Imperialia 3.72-81, del siglo XIII $(=\mathbf{C})^{31}$; esta versión, más cercana a la primera redacción, contiene también secciones de la redacción anglosajona, lo que quiere decir que, probablemente, su modelo presentaba un texto más cercano al original y anterior a la escisión en ambas redacciones.

Como señalaremos con detalle en otro lugar ${ }^{32}$, el texto de la carta es un producto típico de la pseudepigrafía tardoantigua que ha tenido una gran divulgación en el Medievo e influido notablemente en la configuración del imaginario medieval acerca de los mirabilia

S. Mittman, Maps and Monsters in Medieval England, New York-London, Routledge, 2006, pp. 69-81; por su parte, Elisa Ramazzina, «Le Meraviglie d'Oriente: due versioni a confronto», en AA.VV., Medioevi Moderni - Modernità del Medioevo. Saggi per Maria Grazia Saibene, Venezia, Università Ca' Foscari, 2013, pp. 509-524 realiza una comparación de las dos versiones anglosajonas y plantea la hipótesis de que en las supresiones y añadidos subyazca una exégesis alegórico-cristiana.

${ }^{27}$ Es el famoso manuscrito del Beowulf, vid., principalmente, Kenneth Sisam, Studies in the History of Old English Literature, Oxford, Clarendon Press, 1967², pp. 61-96; Andy Orchard, Pride and Prodigies. Studies in the Monsters of the Beowulf-Manuscript, Toronto, University Press, 2003², pp. 1-27; y del mismo A Critical Companion to Beowulf, Cambridge, D.S. Brewer, 2003, pp. 12-56.

${ }^{28}$ Editado por primera vez por Alfons Hilka, «Ein neuer (altfranzösischer) Text des Briefes über die Wunder Asiens», Zeitschrift für französische Sprache und Literatur, 46 (2003), pp. 92-103, el texto se puede encontrar también en la edición citada de Lecouteux y en Knock, quien además dedica las pp. 175-185 a establecer su relación con la versión $\mathbf{D}$.

${ }^{29} \mathrm{El}$ manuscrito se quemó en 1870 , pero afortunadamente fue transcrito por Eberhard G. Graff, Diutiska. Denkmäler deutscher Sprache und Literatur, II, Stuttgart, Cotta, 1827, pp. 194-198, de quien deriva el resto reproducciones.

${ }^{30}$ El texto de la carta fue por primera vez editado en Thomas O. Cockayne, Narratiunculae Anglicae Conscriptae, London, J.R. Smith, 1881, pp. 62-66; Ann E. Knock, Wonders of the East..., op. cit., pp. 65-83 lo denomina Mir y señala, además, la existencia de un apógrafo de la version latina (ms. Bodley 614, siglo XII ${ }^{\text {ex. }}$ ) que estudia con detalle; otra versión de $\mathbf{D}$ en Lecouteux, art. cit., especialmente pp. 104-105 y pp. 129-134, con edición del texto a partir de la transcripción de Johannes B. Pitra, Analecta Sacra spicilegio Solesmensi parata, vol. II, Tusculum, Typis Tusculanis, 1884, pp. 647-649, pues el manuscrito no se ha localizado.

${ }^{31}$ Además de la edición de Gottfried W. Leibnitz, Scriptores Rerum Brunsvicensium, vol. I, Hanoniae, 1707, pp. 984-986 (para la carta), tenemos presente la más reciente y crítica de Sheila E. Bank y James W. Binns, Gervase of Tilbury. Oria Imperialia. Recreation for an Emperor, Oxford, Clarendon Press, 2002; véase el minucioso análisis comparativo de Ann E. Knock, Wonders of the East..., op. cit., pp. 261-298 y un estudio del modus operandi de Gervasio en Fortunata Latella, «Come lavorava un intellettuale laico del Medioevo. Gli Otia Imperialia di Gervasio di Tilbury tra inuentio e compilatio», Revista de Literatura Medieval, 25 (2013), pp. 103-134.

${ }^{32}$ En la introducción de la edición bilingüe y anotada del manuscrito parisino que esperamos ver pronto publicada. 
Orientalia $^{33}$. En esta ocasión, presentamos un estudio pormenorizado de la versión epitomada que contiene el ms. 19 de la Biblioteca Nacional, editando por primera vez el texto latino en confrontación con las otras recensiones del mismo grupo y de la epistula en general.

\section{ANÁLISIS PALEOGRÁFICO}

En $\mathbf{A}^{3}$ el texto de la Epistula se halla en fols. 198r-199r, copiado inmediatamente después del precedente (Liber Iunoris Philosophi in quo continetur totius mundi descriptio, fols. 195r-198r). El cambio de obra se efectúa mediante dos habituales recursos distintivos ${ }^{34}$ : el uso de tinta roja -que esporádicamente será empleada también en las iniciales de cada capitula de la carta, pero casi de forma sistemática en el resto del manuscrito- y una capital inicial de tamaño solemne ${ }^{35}$. No hay ningún tipo de división en capitula, ni intertítulos que seccionen el texto: solo las capitales pueden orientar para la estructura de la carta, escritas en tamaño común, pero en posición destacada, cuando coinciden a principio de línea (solo en 4 ocasiones).

A nivel de palabra, se aprecia una clara intención por evitar el corte al final de línea ${ }^{36}$, con solo un $23 \%$ de palabras cortadas, de las cuales un $69 \%$ presenta el guión de corte; el copista, por tanto, prefiere no interrumpir la lectura y para ello utiliza a final de línea algún tipo de abreviatura (59\% de los casos).

En cuanto al sistema abreviativo empleado, es el habitual en este tipo de escritura:

a. Abreviaturas por suspensión: mediante los signos abreviativos comunes para las sílabas finales, es decir, $-=-m$, $-n t$, -men (también en interior de palabra: p. ej. pauimento), -er (también en interior de palabra: p. ej. terram), -ut (caput, aput), -b; =-bus, ${ }^{\circ}=-u s$,

${ }^{33}$ Ya lo señaló Rudolf Wittkower, «Marvels of the East. A Study of the History of Monsters», Journal of the Warburg and Courtauld Institutes, 5 (1942), pp. 159-197, estudio fundamental sobre las representaciones iconográficas; véanse también los trabajos de Patrizia Lendinara, «Popoli fantastici nei Bestiari», en Fulvio Ferrari y Massimiliano Bampi (eds.), Le lingue e le letterature germaniche fra il XII e il XVI secolo. Atti del XXIX Convegno dell'Associazione Italiana di Filologia Germanica (Trento 5-7 giugno 2002), Trento, Università di Trento, 2004, pp. 303-334 y «Rileggere e schedare i portenti», en Carmela Rizzo (ed.), Fabelwesen, mostri e portenti nell'immaginario occidentale. Medioevo germanico e altro, Alessandria, Edizioni dell'Orso, 2004, pp. 237-280.

${ }^{34}$ Sobre la utilización de recursos publicitarios y distintivos en la documentación latina vid. Vicente García Lobo, Los medios de comunicación social en la Edad Media. La comunicación publicitaria. Lección inaugural curso académico 1991-92, León, Universidad de León, 1991.

${ }^{35}$ De acuerdo con la tipología propuesta por María Encarnación Martín López, «La escritura publicitaria», en AA.VV., Codex Biblicus Legionensis. Veinte estudios, León, Universidad de León, 1999, pp. 127-140.

${ }^{36}$ Un estudio sobre el fenómeno en los códices griegos en Marilena Maniaci, «Alla fine della riga. Divisione delle parole e continuità del testo nel manoscritto bizantino», Scriptorium, 51 (1997), pp. 189-233. 
«2-symbol» $=-u r(\text { también en interior de palabra: p.ej. natura })^{37}$, $-4=-r u m$. Interesante es la mayoritaria proporción de formas sin abreviar del verbo sum, frente a las comunes abreviaturas $\overline{\mathrm{e}} \mathrm{y} \mathrm{st}^{\prime}$ para est y sunt respectivamente, con unos porcentajes muy similares: est $64 \%$ :: $\overline{\mathrm{e}} 36 \%$ / sunt $60 \%$ :: st' $40 \%$. También es llamativo el uso de una -s en voladito: eos (fol. 198v-a.44), his (fol. 198v-b.12, pero ante correctionem), scimindris (fol. 199r-a.5).

b. Abreviaturas por contracción: no son muy frecuentes, reducidas prácticamente a formas personales y participiales del verbo habeo ( $\mathrm{ht}=$ habet, $\mathrm{hbnt}=$ habent, hntes $=$ habentes, $\widehat{\mathrm{hn}} \mathrm{s}=$ habens $)$, diferentes casos del nomen sacrum homo, hominis (hôem = hominem, hoies $=$ homines, hoib; $=$ hominibus) y a términos como octos $=$ oculos, amal = animal, etc.

c. Ligaduras: $\&=$ et (también a final del palabra: p. ej. manet) y -st-, con extensión del brazo de s alta (I) hasta la t que ya no tiene forma de tau $(\tau)$. No hay ejemplos de ae dado que se utiliza la $e$ caudata para anotar la e procedente el antiguo diptongo clásico, si bien, como veremos, el copista yerra en algunas ocasiones. Solo se constata un caso de ligadura -or: nitor (fol. 199r-a.17).

A nivel textual, la copia está bastante limpia, pero ha sido corregida, creemos, en dos fases:

a. Una primera mano -quizá la manus textualis- ha actuado sobre el texto con ciertas mejoras: adición supra lineam de la preposición ad omitida (con la misma d uncial esporádicamente empleada por el copista) ${ }^{38}$; corrección de regiaque como regia que, borrando el signo abreviativo de q; y añadiendo $u$ y $e$ en voladito con las mismas características gráficas de la manus textualis, lo que implica revisión por parte de copista durante el mismo proceso de transcripción del modelo.

b. Otra mano ha efectuado la mayoría de las correcciones en el texto, realizadas en una fase posterior como se aprecia por el distinto tipo de tinta e, incluso, por la diferente caligrafía; generalmente esta segunda mano sobrescribe sus propuestas y/o indica las letras que deben corregirse con un punto bajo. Las diferencias gráficas con respecto de la manus textualis son evidentes en la forma de $u$ como $v$ (p. ej. bobis a.c./bovis p.c.) y en la abreviatura -bus como -b', no como -b;. Puede que esta misma mano sea la autora de la única nota in margine (fol. 198v): Mirmydones, indicando la localización del capitulum sobre las gigantescas hormigas de la India. Sin embargo, no todas las propuestas son correctas y así, por ejemplo,

${ }^{37}$ Acerca de la evolución en el uso de este signo, en lugar del apóstrofe vid. Edward K. Rand, «On the symbols of abbreviations for-tur», Speculum, 2 (1937), pp. 52-65.

${ }^{38}$ P. ej. ad fol. $198 \mathrm{v}-\mathrm{a} .19$, dentes fol. $198 \mathrm{v}-\mathrm{a} .25$, retardantes fol. $198 \mathrm{v}-\mathrm{a} .44$, tritonides fol. $198 \mathrm{v}-\mathrm{b} .30$, pedes fol. $198 \mathrm{v}-\mathrm{b} .32$ ante correctionem. 
en fol. $198 \mathrm{v}-\mathrm{b} .38$ corrige el original colonia, presente en todas las redacciones, con coluna, quizá por haber leído al sucesión $n i$ como un, factible en este tipo de escritura y suponiendo también un error en la vocal.

c. Es difícil determinar a qué mano corresponden las litterae erasae que no han sido sobrescritas, p. ej.: colore(s) similes (fol. 198v-b.6), claro error por anticipación de f-; (h)os (fol. 198v-b.12), por confusión en el uso de h-; $h a(\mathrm{n}) c$ (fol. 198v-b.44), quizá por perseverancia de la preposición in, etc.

Por lo general, $\mathbf{A}^{3}$ presenta un texto más correcto que los otros testimonios, incluso que el propio $\mathbf{A}$ :

Philonis $\mathbf{A}^{\mathbf{3}}$ : Filonis $\mathbf{A}^{\mathbf{1}}$ formam maximam $\mathbf{A}^{\mathbf{3}}$ : forma maxima $\mathbf{A}^{\mathbf{1}} \mathbf{A}^{\mathbf{4}}$ onagri $\mathbf{A}^{\mathbf{3}}$ : honagri $\mathbf{A}^{\mathbf{4}}$ Arabiam $\mathbf{A}^{\mathbf{3}}$ : Arabyam $\mathbf{A}^{\mathbf{2}}$ cerastes $\mathbf{A}^{\mathbf{3}}$ : caraste $\mathbf{A}$, cerastes $\mathbf{A}^{\mathbf{2}}$ hominibus $\mathbf{A}^{\mathbf{3}}$ : ominibus $\mathbf{A}^{2} \quad c y$ nocephali $\mathbf{A}^{\mathbf{3}}$ : quinocoephali $\mathbf{A}$, cunocephali $\mathbf{A}^{\mathbf{1}}$ cursu $\mathbf{A}^{\mathbf{3}}$ : occursu $\mathbf{A}^{\mathbf{2}}$ usque ad horam $\mathbf{A}^{\text {3p.c. }}$ : usque in ora $\mathbf{A}$, usque hora cett. codd. transeunt flumen $\mathbf{A}^{3}$ : transeunt in flumen $\mathbf{A}^{4}$ feminis $\mathbf{A}^{3}$ : fenminis $\mathbf{A}^{4}$ Brisonem $\mathbf{A}^{\mathbf{3}}$ : Brysonem $\mathbf{A}^{\mathbf{4}}$ Nilum $\mathbf{A}^{\mathbf{3}}$ : Nylum $\mathbf{A}^{\mathbf{2}} \mathbf{A}^{\mathbf{4}}$ elephantorum $\mathbf{A}^{\mathbf{3}}$ : elefantorum $\mathbf{A A}^{\mathbf{1}}$ caput $\mathbf{A}^{\mathbf{3}}$ : capite $\mathbf{A}^{4}$ caput magnum $\mathbf{A}^{3}$ : capud magno $\mathbf{A}^{4}$ stadiis $\mathbf{A}^{3}$ : stadus $\mathbf{A}^{\mathbf{1}} \quad$ cincta $\mathbf{A}^{\mathbf{3}}$ : cinta $\mathbf{A}^{\mathbf{4}}$ thus $\mathbf{A}^{\mathbf{3}}$ : tus $\mathbf{A}^{\mathbf{1}}$ opobalsamum $\mathbf{A}^{\mathbf{3}}$ : apobalsamum $\mathbf{A}$ loco $\mathbf{A}^{\mathbf{3}}$ : om. $\mathbf{A}^{\mathbf{1}}$ arula $\mathbf{A}^{3}$ : trula $\mathbf{A}^{\mathbf{4}}$ et longitudine $\mathbf{A}^{3}$ : om. $\mathbf{A}^{4}$ est $\mathbf{A}^{3}$ : om. $\mathbf{A}^{4}$ pendent $\mathbf{A}^{3}$ : pendunt $\mathbf{A}^{4}$ thus $\mathbf{A}^{3}$ : tus $\mathbf{A}^{\mathbf{1}}$ caput $\mathbf{A}^{\mathbf{3}}$ : capud $\mathbf{A}^{\mathbf{4}}$ uiuat $\mathbf{A}^{\mathbf{3}}$ : uiuet $\mathbf{A}$, bibat $\mathbf{A}^{\mathbf{1}}$

No obstante, también contiene errores, generalmente de tipo ortográfico:

Babiloniam $\mathbf{A}^{\mathbf{3}}$ : Babyloniam $\mathbf{A}^{\mathbf{1}} \mathbf{A}^{\mathbf{4}}$ orridi $\mathbf{A}^{\mathbf{3}}$ : horridi $\mathbf{A} \mathbf{A}^{\mathbf{1}} \mathbf{A}^{\mathbf{4}}$ igne $\mathbf{A}^{\mathbf{3}}$ : igne cett. codd. ęuntibus $\mathbf{A}^{\mathbf{3}}$ : euntibus cett. codd. mirmidones $\mathbf{A}^{\mathbf{3}}$ : mirmidones $\mathbf{A A}^{1}$ reliqunt $\mathbf{A}^{\mathbf{3}}$ : relinquunt cett. codd. Egiptus $\mathbf{A}^{\mathbf{3}}$ : Egyptus $\mathbf{A}^{2} \mathbf{A}^{4}$ reliquum $\mathbf{A}^{3}$ : reliquum cett. codd. brachia $\mathbf{A}^{3}$ : bracchia $\mathbf{A}^{4}$ extructa $\mathbf{A}^{3}$ : exstructa $\mathbf{A}^{4} \quad$ LX $\mathbf{A}^{3}$ : LXX cett. codd. sicimindris $\mathbf{A}^{3}$ : scinindris $\mathbf{A}^{\mathbf{1}} \mathbf{A}^{\mathbf{4}}$

Por otra parte, parece que el modelo común del grupo transmitió varias corruptelas que han dado lugar a lecturas discordantes, sobre todo en nombres de origen griego como ictiófagos, hipopótamos, fénix o sóraces (cfr. infra).

Finalmente, $\mathbf{A}^{3}$, al igual que $\mathbf{A}^{2}$ y $\mathbf{A}^{4}$, presenta una interpolación procedente de Solino que implica la existencia de un modelo común para los tres ${ }^{39}$.

\footnotetext{
${ }^{39}$ Solin. XXXIII 11.
} 


\section{CRITERIOS DE EDICIÓN}

Ofrecemos a continuación la única edición, que conozcamos ${ }^{40}$, de esta recensión de la carta, basada en la lectura del ms. a través de la excelente digitalización que ofrece la BNM, cotejando también las otras redacciones del mismo grupo a partir de las transcripciones realizadas por los estudiosos precedentes y de nuestra propia lectura de los manuscritos parisinos. Para ello, siguiendo la tónica iniciada por Omont y seguida por Faral y Lecouteux, utilizamos las siglas ya presentadas supra para las versiones del mismo grupo, es decir:
$\mathbf{A}=$ Parisinus NAL 1065, fols. $92 \mathrm{v}-95 \mathrm{v}$
$\mathbf{A}^{\mathbf{1}}=$ Casinensis 391 , fols. $82 \mathrm{v}-84 \mathrm{v}$
$\mathbf{A}^{2}=$ Cauensis 3, fols. 397r-398r
$\mathbf{A}^{3}=$ Matritensis 19, fols. 198r-199r
$\mathbf{A}^{4}=$ Parisinus AFL 7418, fols. 268r-270v

Asimismo, para poder localizar y comparar la versión de nuestro texto con la del resto de versiones, señalamos las correspondencias con el sistema de párrafos de la edición de Faral.

Aunque la carta en sí presenta en ambas redacciones infinidad de incoherencias sintácticas y morfológicas que han escapado a los estudiosos precedentes, en su mayoría no latinistas ${ }^{41}$, por nuestra parte solo vamos a actuar sobre el texto del manuscrito madrileño cuando muestra errores flagrantes (generalmente confusiones ortográficas) y lecturas heredadas del arquetipo de toda la familia, como, por ejemplo, centrios A : centrias $\mathbf{C}$, cintras cett. codd. Aunque Gervasio podría conservar el texto correcto, ofrece, sin embargo, la explicación errónea id est medias partes, que es como precisamente se ha interpretado en el resto de testimonios; no obstante, creemos que la

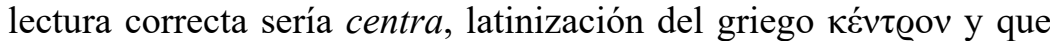
en terminología zoológica admite el significado de «espina» ${ }^{42}$, muy apropiado para las locuste marine del texto; lo mismo cabría decir del ornitónimo strutio et camelus, simplificado por $\mathbf{C}$ ut camelus, que, sin duda, figuraría en el original con la forma latina struthocamelus, del griego $\sigma \tau \rho \circ v \theta$ oкó $\mu \eta \lambda \varsigma^{43}$. Otro caso significativo es la

${ }^{40}$ Ann E. Knock, Wonders of the East..., op. cit., pp. 933-940 solo ofrece en notas las variantes de $\mathbf{A}^{\mathbf{3}}$ con respecto de $\mathbf{A}^{\mathbf{1}}$, aunque en ocasiones yerra en sus lecturas, cfr. infra app.

${ }^{41}$ El texto que editaremos de la versión de A diferirá, por tanto, del resto de ediciones precedente porque pretendemos llevar a cabo una edición crítica real que contemple las posibles deformaciones sufridas por la epístola desde su composición en la Antigüedad Tardía hasta su circulación medieval, teniendo en cuenta la previa escisión en las dos redacción y todas las alteraciones recibidas durante el proceso de reducción al que ha sido sometida.

${ }^{42}$ Cfr. ThLL s.v. centrum (4).

${ }^{43}$ Vid. Jacques André, Les noms d'oiseaux en latin, Paris, Klincksieck, 1967, p. 147 y F. Capponi, Ornithologia latina, Genova, Istituto di Filologia Classica e Medievale, 1979, pp. 470-472. 
lectura lupe o luppe de todos los miembros de esta redacción, mientras que los testimonios de la otra recensión dan gripes $\mathbf{B}$ o griphus D; más que suponer una lectura upupe ${ }^{44}$, creemos que en el original se estaba comparando el plumaje del ave prodigiosa descrita con el no menos maravilloso grifo, híbrido águila-león de origen oriental que ya figura en frescos cretomicénicos ${ }^{45}$, por lo que el arquetipo de esta redacción estaba corrupto y había deformado la latinización gryps, grypis, de $\gamma \rho \tilde{\psi} \psi, \gamma \rho v \pi \varsigma^{46}$.

Así, en cuanto a los grecismos, hemos optado por transcribirlos según la norma clásica, a fin de evitar confusiones: Babyloniam : Babiloniam $\mathbf{A}^{\mathbf{2}} \mathbf{A}^{3}$; Ichthyophagi : Idtofagi $\mathbf{A}$, Indiophagi $\mathbf{A}^{1}$, Ithiophalii $\mathbf{A}^{2}$, Ithiogi $\mathbf{A}^{\mathbf{3}}$, Eithiophagi $\mathbf{A}^{4}$; myrmidones : mirmidones $\mathbf{A}^{3}$, mirmydones $\mathbf{A}^{\mathbf{3 m a r g} .}$ ex alia manu; Egyptus : Egiptus $\mathbf{A}^{\mathbf{1}} \mathbf{A}^{\mathbf{3}}$; hippopotamus : yppofogi $\mathbf{A}$, yppophagi $\mathbf{A}^{\mathbf{1}} \mathbf{A}^{\mathbf{4}}$, ippophagi $\mathbf{A}^{\mathbf{2}}$, rippophagino $\mathbf{A}^{\mathbf{3}}$; Soraci : Orhaci $\mathbf{A}^{\mathbf{1}} \mathbf{A}^{\mathbf{2}}$, Orbaci $\mathbf{A}^{\mathbf{3}} \mathbf{A}^{\mathbf{4}} ;$, Heliopolis : Eliopolis $\mathbf{A}^{\mathbf{3}}$, Phoenix : Phenix A, Fenix cett. codd. Si, como creemos, la utilización de helenismos corresponde al recurso retórico de la barbarolexis ${ }^{47}$ y no es indicio categórico para sostener la hipótesis de que la carta original estuviera escrita en griego ${ }^{48}$, habría también que actuar en términos como thus, frente al tus de algunas versiones, en tanto que transcripción del griego $\theta v ́ o \varsigma$ y que ya en latín clásico se generaliza tus ${ }^{49}$.

Recibido: $13 / 12 / 2017$

Aceptado: 21/5/2018

${ }^{44}$ Como piensan Bank y Binns, op. cit., p. 707, n. 2.

${ }^{45}$ Vid. Hugo Prinz y Konrat Ziegler, «Gryps», RE, VII.2 (1912), cols. 1902-1929. Cuanto menos curiosa es la racionalización moderna de Adrienne Mayor, The First Fossil Hunters. Paleontology in Greek and Roman Times, Princeton, University Press, 2000, pp. 15-53, que ve detrás de la imagen del grifo algún avistamiento de fósiles de Protoceratópsidos.

${ }^{46}$ Vid. André, op. cit., pp. 89-90, Capponi, op. cit., p. 287.

${ }^{47}$ Cfr. Heinrich Lausberg, Manual de retórica literaria, vol. II, trad. esp. Madrid, Gredos, 1967, pp. 23-24.

${ }^{48}$ Idea ya emitida por Max Förster, «Zur altenglischen Mirabilien-Version», Archiv für das Studium der neueren Sprachen und Literaturen, 117 (1906), pp. 367-370, que ha sido repetida y justificada, entre otros, por Ann E. Knock, Wonders of the East..., op. cit., pp. 25-29 o Giuseppe Tardiola, Le meraviglie dell'India, Roma, Archivio Guido Izzi, 1991, p. 41, pero, como decimos, no hay indicios suficientes y puede que, en realidad, todo forme parte de la falsificación realizada por el autor de la carta.

${ }^{49}$ Cfr. Jacques André, Lexique des termes de botanique en latin, Paris, Klincksieck, 1956, pp. 228-229. 


\title{
$\cos$
}

\section{BNM MS. 19 (OLIM A 16): MIRABILIA ORIENTALIA INTRODUCCIÓN, EDICIÓN Y TRADUCCIÓN}

\begin{abstract}
RESUMEN: El ms. 19 de la BNM conserva una versión epitomada de la carta sobre los mirabilia Orientalia, epístola pseudepígrafa de gran importancia para la tradición de las maravillas de Oriente durante el Medievo y el Renacimiento, conservada en dos redacciones diferentes y en varias versiones epitomadas. Aunque hay ediciones sinópticas de las principales versiones de ambas redacciones, el texto del ms. 19 de la BNM sigue inédito. En este trabajo editamos y traducimos por primera vez la versión Matritensis de la carta en confrontación con el resto de versiones de la misma redacción.
\end{abstract}

Palabras ClaVE: Mirabilia Orientalia, edición crítica, monstruos y maravillas, Medievo.

\section{BNM MS. 19 (OLIM A 16): MIRABILIA ORIENTALIA} INTRODUCTION, EDITION AND TRANSLATION

ABSTRACT: Ms. 19 from BNM contains a brief version of the famous letter about Mirabilia Orientalia, a pseudepigraphic epistle crucial for the tradition of the monsters and Orient marvels during the Middle Ages and the Renaissance; it has been preserved in two different redactions and in several manuscript versions. In this paper we present for the first time an edition and translation of the version of the codex Matritensis.

Keywords: Mirabilia Orientalia, critical edition, monsters and marvels, Middle Ages. 
Feramen Rex ad Adrianum Imperatorem ${ }^{50}$

$<$ VII-X.> Inter Babyloniam et Mesopotamiam est regio locuples, in qua est oppidum quod dicitur Castellum Philonis, ubi nascuntur humiles bestiolę quasi simię. Quando sonum audiunt, fugiunt, pedes habentes octonos idemque et oculos, cornua bina habentes. Quas cum aliquis occidere uoluerit, diligenter se munire debet. ${ }^{51}$

$<$ XI.1. $>$ Est et alia regio, quę dicitur Pelusia, distans a Babylonia stadia $\left.\right|^{198 v}$ sexaginta, ubi serpentes nascuntur immensi atque horridi et nimis seuissimi, habentes bina capita, quorum oculi lucent ut lucerna. $<2$. $>$ Nascuntur ibi et onagri cornua habentes et formam maximam..$^{52}$

$<$ XII. $>$ Hec iuxta Arabiam, a Mari Rubro pergens in Arabiam est regio inculta propter serpentes. In quibus locis nascuntur serpentes cerastes, cornua similia habentes arietum; hominem cum percusserint, occident. Ibi nascitur et multitudo piperis, quod idem serpentes custodiunt. Quod sic ab hominibus colligitur: cum maturum fuerit, incendunt eadem loca et serpentes ab igne fugantur et se sub terram mittunt. Quapropter piper nigrum efficitur, nam natura album est. ${ }^{53}$

$<$ XIV.> Seleucia a parte dextra euntibus ad Mare Rubrum sunt uicinales diuisiones. Ibi nascuntur Cynocephali, iubam equorum habentes ualidissimis atque immensis dentibus flammas spirantes. ${ }^{54}$

$<$ XVI.1. $>$ Hinc non longe abest insula in qua nascuntur homines longi, habentes barbam usque ad genua, qui appellantur Ichthyophagi; pisces crudos uescuntur. ${ }^{55}<2$. $>$ Est enim fluuius in eadem insula nomine Gargarus; trans hoc flumen nascuntur formicę myrmidones, magnitudine catulorum, habentes pedes senos et centra quasi locustę marinę, dentes habentes canum; colore autem sunt nigrę aurumque fodiunt et proferunt de subterraneo ad lucem; quę cum hominem uel aliud animal conspexerint, deuorant usque ad ossa; sunt enim uelocissime in cursu, ut putes eas uolare. Hę Sole Oriente usque ad horam quintam sub terram sunt et aurum fodiunt, quod ex ingenio hominum qui ibidem sunt confines ita aufertur: inducunt camelos et feminas cum polledris suis, quos polledros super ripam fluminis relinquunt ligatos et ipsi transeunt flumen cum camelis masculis et

\footnotetext{
${ }^{50}$ Tit.: Feramen $\mathbf{A}^{3}$ : Fermes A, om. cett. codd.

${ }^{51}<$ VII-X. $>$ Babyloniam scrip. ex $\mathbf{A}^{1} \mathbf{A}^{4}$ : Babiloniam $\mathbf{A}^{2} \mathbf{A}^{3} \quad$ locuples scrip. ex $\mathbf{A}^{1} \mathbf{A}^{2}$ : locuplex $\mathbf{A}^{3} \mathbf{A}^{4}$

${ }_{52}^{52}<$ XI.> Babylonia scrip. ex pler. codd. : Babilonia $\mathbf{A}^{3} \quad$ horridi scrip. ex $\mathbf{A}^{1} \mathbf{A}^{4}$ : orridi $\mathbf{A}^{2} \mathbf{A}^{3}$

${ }^{53}<$ XII.> igne scrip. ex pler. codd. : ignę $\mathbf{A}^{3}$

${ }^{54}<$ XIV.> euntibus scrip. ex pler. codd. : ęuntibus $\mathbf{A}^{\mathbf{3}}$ iubam scrip. ex $\mathbf{A}^{\mathbf{1}}$ : iuba cett. codd.

$55<$ XVI.1 > Ichthyophagi scrip. : Idtofagi $\mathbf{A}$, Indiophagi $\mathbf{A}^{\mathbf{1}}$, Ithiophalii $\mathbf{A}^{\mathbf{2}}$, Ithiogi $\mathbf{A}^{\mathbf{3}}$, Eithiophagi $\mathbf{A}^{\mathbf{4}}<2$.> insula $\mathbf{A}^{\text {3p.c. }}$ (signum eras.) : insulam $\mathbf{A}^{\text {3a.c. }}$ (ut $\mathbf{A}^{\mathbf{2}}$ ) myrmidones scrip. ex $\mathbf{A}^{\mathbf{1}}$ : mirmidones $\mathbf{A}^{3}$, mirmydones $\mathbf{A}^{3 \text { marg. }}$ ex alia manu centra scrip. : cintras pler. codd., centrios A, centrias $\mathbf{C}$ ad add. s.l., om. cett. codd. polledris scrip. : pulletris $\mathbf{A}$, pullis $\mathbf{A}^{\mathbf{1}}$, polletris cett. codd.
} 


\section{El rey Feramen al emperador Adriano}

$<$ VII-X. $>$ Entre Babilonia y Mesopotamia hay una opulenta región en la que se halla la plaza fuerte que se llama Castillo Filonis, donde se crían unas bestezuelas asustadizas similares a monas. Cuando oyen un ruido, salen huyendo; tienen ocho patas y los mismos ojos, y dos cuernos. Quien quiera matarlas, debe equiparse a conciencia.

$<$ XI.1. $>$ Hay también otra región a sesenta estadios de Babilonia que se llama Pelusia, donde se crían enormes y espantosas serpientes, sin duda las más peligrosas, pues tiene dos cabezas y sus ojos brillan como una lámpara. $<2$. $>$ Se crían allí también onagros que tienen cuernos y una mayor envergadura.

$<$ XII. $>$ Una región junto a Arabia, yendo desde el Mar Rojo hacia Arabia, es estéril a causa de las serpientes: en estos parajes se crían las serpientes cerastas, que tienen cuernos parecidos a los de los carneros; matarán al hombre al que piquen. Allí se da también abundancia de pimienta, a la que custodian las mismas serpientes. Así es recolectada por los hombres: cuando está madura, prenden fuego a esos lugares y las serpientes huyen del fuego y se esconden bajo tierra. Por esto la pimienta se pone negra, pues por naturaleza es blanca.

$<$ XIV. $>$ En Seleucia, yendo por la parte derecha hacia el Mar Rojo, hay distritos vecinales. Allí se cían los cinocéfalos, que tienen crines de caballo, con poderosísimos e inmensos dientes y exhalan fuego.

$<$ XVI.1. $>$ No lejos de allí hay una isla en la que nacen unos seres humanos altos, con la barba hasta las rodillas, que se llaman ictiófagos; se alimentan de peces crudos. $<2$. $>$ En esa misma isla está, además, el río Gargaro; al otro lado del río se crían las hormigas mirmídones, del tamaño de cachorros, con seis patas y espinas como las langostas marinas, y dientes de perro; de color son, en cambio, negras y excavan oro y lo sacan a la luz del subsuelo. Cuando se encuentran con un humano o algún otro animal, lo devoran hasta los huesos. Son, además, muy veloces en la carrera, tanto que pensarías que vuelan. Estas, desde el amanecer hasta la hora quinta están bajo tierra y excavan el oro, que es extraído con el ingenio de los hombres que habitan aquellos confines de la siguiente manera: llevan camellos y hembras con sus crías, a las que dejan amarradas en la orilla del

* Añadimos una traducción literal y fiel de la versión matritense de epistula; se ha intentado dar sentido a un remendado texto pleno de errores gramaticales, redundancias y omisiones, que requiere, además, de numerosas notas explicativas sobre el contenido de la carta. Remitimos, por tanto, a nuestra futura edición comentada para cotejar las fuentes, variantes y tradición de tan singular obra. 
feminis. Quippe cum ad locum peruenerunt, camelis feminis aurum imponunt et ille ad filios festinantes suos plus quam cursim fluuium transeunt. Homines uero, dum uiderint formicarum agmina se sequi, relinquunt camelos masculos et fugiunt atque $<\mathrm{ad}>$ fluuium cum camelis feminis uolantes eunt. Formicę autem, cum camelos masculos inuenerint, deuorant eos et retardantes, cum consequi uoluerint, transire non possunt. ${ }^{56}$

$<$ XVII.1-3. $>$ Est inter Brisonem fluuium et Nilum, quo Egyptus irrigatur, regio que dicitur Mesopotamia, in qua est multitudo elephantorum ibique nascuntur homines longa femora habentes duodecim, reliquum uero corpus pedum sex, candida bra $<\mathrm{c}>$ chia usque ad humeros, nigres aures, pedes rubei, caput rotundum, nares longas. $<4$. $>$ Ibique nascuntur bestie colore similes equorum, pedes habentes leonum, altitudine habentes pedes Xxx, grossitudine pedum duodecim. $<$ Hanc bestiam si quispiam persequi uoluerit $>$, harum sanguine aluntur homines; qui hippopotami nominantur. $<5$. $>$ Est namque et alia insula in Brisone fluuio, ubi nascuntur homines sine capite, habentes oculos et os in pectore. hi longi sunt pedibus duodecim, lati et uasti pedes VII, colore et pectore auro similes. $<6$. $>$ Ibidem nascuntur dracones immensi longitudinis, forma pedibus CL grossi, latitudine columnarum maximarum. ${ }^{57}$

$<$ XXI. $>$ Circa Indiam et Arabiam regio est in cuius montibus sunt mulieres horride, habentes barbas usque ad mammas, caput planum, pellibus uestite. Sunt namque uenatrices pro canibus bestias nutriunt ad uenationem; quę bestię magnitudine et colore leonibus comparantur. ${ }^{58}$

$<$ XXII.> In eisdem montibus siluę sunt in quibus nascuntur et alie mulieres aprorum dentes habentes, capillos ad talos et in lumbis caudas bouis altae pedibus XII, reliquum autem corpus pilosum quasi struthocamelus. ${ }^{59}$

$<$ XXV. $>$ In Persarum uero partibus nascuntur Soraci, qui aput nos Tritonides appellantur, quasi diuini. Quos de quacumque causa interrogare uolueris, responsum accipies. ${ }^{60}$

${ }^{56}$ polledros scrip. : pulletros $\mathbf{A}$, pullos $\mathbf{A}^{\mathbf{1}}$, polletros cett. codd. relinquunt scrip. ex cett. codd. : relinqunt $\mathbf{A}^{3}<\mathrm{ad}>$ add. ex $\mathbf{A}^{\mathbf{1}}$, om. cett. codd.

${ }^{57}<$ XVII.1-3.> Egyptus scrip. ex $\mathbf{A}^{\mathbf{2}} \mathbf{A}^{\mathbf{4}}$ : Egiptus $\mathbf{A}^{\mathbf{3}}$ (ut $\left.\mathbf{A}^{\mathbf{1}}\right)$ regio que $\mathbf{A}^{\text {3p.c. }}$ : regiaque $\mathbf{A}^{\text {3a.c. }} \quad$ reliquum scrip. ex cett. codd. : reliqum $\mathbf{A}^{3}$ sex $\mathbf{A}^{3 \text { p.c. }}$ : senum $\mathbf{A}^{1} \mathbf{A}^{2} \mathbf{A}^{\text {3a.c. }}$, sednum $\mathbf{A}^{4} \quad$ nigres aures scrip. ex $\mathbf{A}^{2}$ : nigresurę sic $\mathbf{A}^{3}$, nigre sure $\mathbf{A}^{\mathbf{1}}$ nares $\mathbf{A}^{\text {3p.c. }}$ (n- s.scr.) : cares uel similia $\mathbf{A}^{\text {3a.c. }}<4$.> colore $\mathbf{A}^{\text {3p.c. }}$ (-s eras.) : colores $\mathbf{A}^{\text {3a.c. }}\left(\right.$ ut $\left.\mathbf{A}^{1}\right) \quad<$ hanc...uoluerit> om. $\mathbf{A}^{\mathbf{3}}$ harum $\mathbf{A}^{\text {3p.c. }}$ : arum $\mathbf{A}^{\text {3a.c. }}$ (ut. $\mathbf{A}^{\mathbf{1}}$ ) hippopotami scrip. : yppofogi $\mathbf{A}$, yppophagi $\mathbf{A}^{\mathbf{1}} \mathbf{A}^{\mathbf{4}}$,

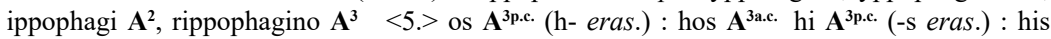
$\mathbf{A}^{\text {3a.c. }}$ pedibus $\mathbf{A}^{\text {3p.c. }}$ (-edes eras. et -ibus s.scr.) : pedes $\mathbf{A}^{\text {3a.c. }}$ pedibus $\mathbf{A}^{\text {3p.c. }}$ (-edes eras. et -ibus s.scr.) : pedes $\mathbf{A}^{\text {3a.c. }}$

${ }^{58}<$ XXI. $>$ horride scrip. : orride $\mathbf{A}^{\mathbf{3}}$ uenatrices sic recte $\mathbf{A}^{\mathbf{3}}$ : benatrices legit Knock

${ }^{59}<$ XXII. $>$ bouis $\mathbf{A}^{\text {3p.c. }}(-\mathrm{u}-$ s.scr. $)$ : bobis $\mathbf{A}^{\text {3a.c. }} \quad$ altae $\mathbf{A}^{\text {3p.c. }}$ (-e- $\left.s . s c r.\right)$ : altas $\mathbf{A}^{\text {3a.c. }}$ pedibus $\mathbf{A}^{\text {3p.c. }}$ (-edes eras. et -ibus s.scr.) : pedes $\mathbf{A}^{\text {3a.c. }} \quad$ struthocamelus scrip. : strutio et camelus $\mathbf{A}^{3}$

${ }^{60}<\mathrm{XXV}$. $>$ Soraci scrip. ex $\mathbf{A C}$ : Orhaci $\mathbf{A}^{\mathbf{1}} \mathbf{A}^{\mathbf{2}}$, Orbaci $\mathbf{A}^{\mathbf{3}} \mathbf{A}^{\mathbf{4}}$ 
río, mientras ellos cruzan el río con los camellos machos y hembras. Cuando llegan al lugar, cargan el oro en las camellas y estas cruzan el río junto a sus crías corriendo más que al galope; entonces los hombres, cuando ven que el ejército de hormigas los persigue, abandonan a los camellos, huyen y salen volando hacia el río con las camellas. Las hormigas, al toparse con los camellos, los devoran y se entretienen, no pudiendo cruzar aunque quieran hacerlo.

$<$ XVII.1-3. $>$ Hay entre el río Brisón y el Nilo, el que riega Egipto, una región que se llama Mesopotamia. En ella hay multitud de elefantes y también allí nacen hombres que tienen largos fémures de doce pies, pero el resto del cuerpo de seis pies, blancos brazos hasta los hombros, negras orejas, pies rojos, cabeza redonda y largas narices. $<4$. $>$ Allí se crían bestias parecidas en el color a los caballos, que tienen patas de león, con una altitud de 30 pies y doce de ancho. $<$ Si alguien quisiera perseguirlas $>$, los hombres se alimentan de su sangre; son llamados hipopótamos. $<5$. $>$ Hay, también, otra isla en el río Brisón, donde nacen hombres in cabeza que tienen los ojos y la boca en el pecho. Estos son de doce pies de altos, anchos y gordos de 7 pies, de color y en el pecho parecidos al oro. $<6$. $>$ Allí mismo se crían serpientes enormes de longitud, gordas de 150 pies, con la envergadura de las más grandes columnas.

$<$ XXI. $>$ Cerca de la Indica y de Arabia hay una región en cuyas montañas hay unas espantosas mujeres que tienen barba hasta los pechos, la cabeza plana y están vestidas con pieles. Como son cazadoras, crían bestias en lugar de perros para la caza; estas bestias son comparables a leones en grandeza y color.

$<$ XXII. $>$ En esas mismas montañas hay bosques en los que nacen también otras mujeres que tienen dientes de jabalí, los cabellos hasta los tobillos y colas de buey en el lomo, de 12 pies de altas, pero el resto del cuerpo peludo como un avestruz.

$<\mathrm{XXV}$. $>$ Ciertamente, en zonas de los persas nace los Sóraces, quienes entre nosotros se llaman Tritonides, son como adivinos; si quisieras preguntarles sobre cualquier cosa, recibirías un oráculo. 
$<$ XXVI.4. $>$ Ibi homines staturam habent pedibus XVI, latitudinem pedum VII, caput magnum habentes, auriculas uero quasi statura similes, corpore candido. Qui cum homines uiderint, auriculas suas extendunt ut putes eos uolare. ${ }^{61}$

$<$ XXVII.1. $>$ In eodem loco insula est habens longitudine et latitudinem stadiis CC in qua colonia est Solis nomine Heliopolis, muro cincta, structa ere et ferro. In quo loco arbores sunt similes lauro et oliue, ex quibus thus et opobalsamum nascitur. $<2$. $>$ In eodem loco sunt ędes duę similes, una ex auro quadrata extructa, alia eręa; in hac ede est arula ex margaritis et scinindris structa, latitudine et longitudine pedum LXx. Est item in eodem loco lectulus Solis ex ebore ${ }^{199 r}$ factus et auro purissimo, lapidibus ornatus pretiosissimis, fulgora eius, id est radios, pedum XVI. Est in eadem ęde uinea aurea, hoc est, uitis ex auro facta atque infixa, cuius pauimentum est ex lapidibus pretiosis et scinindris, in qua pendent racemi ex margaritis et unionibus. Edes uero sacerdotis est ex argento facta; qui sacerdos thus uescitur et opobalsamum bibit, dormit in eodem pauimento sub eadem uinea. ${ }^{62}$

$<$ XXVIII.1.> Circa eam uero mons est adamans inaccesibilis; in quo monte est auis habens caput aquilinum, pennas maximas, similis grypi. <2.> In eodem monte est aue Phoenix ampla, que habet in capite cristam similem pauonis [et fauces cristatas. circa collum fulgore aureo scribitur, postera parte purpureus, extra caudam roseis pennis, in qua ceruleus scribitur nitor]. uiuit annos innumerabiles, nidum habens ex margaritis et unionibus, manet semper in cinnamo. Quid uero uescatur uel quemadmodum uiuat, ignoratur. Ipsa autem nidum suum seseque incendit et ex eisdem cineribus iterum renascitur. Ita fit ut, dum semper moritur, semper uiuat. ${ }^{63}$

${ }^{61}<$ XXVI.4.> pedibus $\mathbf{A}^{\text {3p.c. }}$ (-edes eras. et -ibus s.scr.) : pedes $\mathbf{A}^{\text {3a.c. }} \quad$ pedum $\mathbf{A}^{\text {3p.c. }}$ (-umadd. s.l. et signum sub delendis litteris) : pedes $\mathbf{A}^{\text {3a.c. }}$

${ }^{62}<$ XXVII.1. $>$ colonia recte habet $\mathbf{A}^{\text {3a.c. }}$ : coluna $\mathbf{A}^{\text {3p.c. }}$ (-oni- eras. et $-\mathbf{u}-$ s.l.) Heliopolis scrip. : Eliopolis $\mathbf{A}^{3}$ quo recte $\mathbf{A}^{3}$ : qua legit Knock grypi scrip. : luppe $\mathbf{A}$, lupe cett. codd. $\quad<2 .>$ Hac $\mathbf{A}^{\text {3p.c. }}$ (-n- eras.) : hanc $\mathbf{A}^{\text {3a.c. }} \quad$ LXX scrip. ut cett. codd. : LX $\mathbf{A}^{3}$ pedum $\mathbf{A}^{\text {3p.c. }}$ (-um- add. s.l. et signum sub delendis litteris) : pedes $\mathbf{A}^{\text {3a.c. }}$, pedibus legit Knock est iterauit $\mathbf{A}^{\mathbf{3}}$, sed erasit $\mathbf{A}^{3 \text { p.c. }} \quad$ in eadem iterauit $\mathbf{A}^{3}$, sed erasit $\mathbf{A}^{\text {3p.c. }} \quad$ scinindris scrip. ut $\mathbf{A}^{\mathbf{1}} \mathbf{A}^{\mathbf{4}}$ : scimindris $\mathbf{A}^{2} \mathbf{A}^{3} \quad$ racemi $\mathbf{A}^{\text {3p.c.. }}$ rademi uel similia $\mathbf{A}^{\text {3a.c. }} \quad$ opobalsamum $\mathbf{A}^{\text {3p.c. }}$ (-um- eras. et -o- s.scr.) : opumbalsamum $\mathbf{A}^{\text {3a.c. }}$

${ }^{63}<$ XXVIII.1. $>$ similis $\mathbf{A}^{\text {3p.c. }}$ (-is- s.scr.) : simile $\mathbf{A}^{\text {3a.c. }} \quad<2 .>$ Phoenix scrip. : Phenix $\mathbf{A}$, Fenix cett. codd. ampla que $\mathbf{A}^{\text {3p.c. }}$ (add. -u- s.scr. et -e- s.l.) : amplaque $\mathbf{A}^{\text {3a.c. }} \quad$ [et ... nitor] interpolatum ex Solin. XxxIII 11 in $\mathbf{A}^{2} \mathbf{A}^{3} \mathbf{A}^{4} \quad$ scribitur add. $\mathbf{A}^{\text {3p.c. }}$ s.l. quemadmodum scrip. ut cett. codd. : que amo dum $\mathbf{A}^{3}$ uiuat recte $\mathbf{A}^{3}$ (uiuet legit Knock) : bibat $\mathbf{A}^{\mathbf{1}}$ 
$<$ XXVI.4.> Allí los hombres tienen una estatura de 16 pies y 7 pies de ancho, una gran cabeza y orejas como su estatura, con el cuerpo blanco. Cuando ven a los humanos, extienden sus orejas tanto que pensarás que vuelan.

$<$ XXVII.1. $>$ En ese mismo lugar hay una isla que tiene longitud y anchura de 200 estadios, en la que hay una colonia del Sol de nombre Heliópolis, rodeada por un muro y construida con bronce e hierro. En este lugar hay árboles parecidos al laurel y al olivo, de los que nace el incienso y el opobálsamo. $<2$. $>$ En este mismo lugar hay dos templos parecidos, uno cuadrado, construido en oro, el otro es broncíneo; en este templo hay un altarcito hecho de perlas y cilindros, de una anchura y altura de 70 pies. También está en este lugar el lecho del Sol, hecho de marfil y del más puro oro, adornado con las piedras más preciosas, y un resplandor, es decir, unos rayos de 16 pies. En este mismo tempo hay una viña de oro, es decir, una vid hecha de oro y plantada, cuyo suelo es de piedras preciosas y cilindros, de la que cuelgan racimos de perlas y uniones. Por su parte, el templo del sacerdote está hecho de plata; este sacerdote come incienso y bebe opobálsamo, y duerme en el mismo suelo bajo la viña.

$<$ XXVIII.1. $>$ Cerca de esta, además, está el inaccesible monte Adamans; en él hay un ave con cabeza de águila, las más grandes plumas, parecidas a las del grifo. $<2$. $>$ En ese mismo monte se halla el magnífico ave Fénix, que tiene en la cabeza una cresta parecida a la del pavo real [y la papada encrespada. Se describe con un brillo dorado entorno al cuello, purpúreo en la parte trasera, excepto la cola, con plumas rosáceas, donde se percibe un candor azul]. Vive innumerables años, con un nido de perlas y uniones y permanece siempre en el cinamomo. De qué se alimenta o cómo vive, se desconoce. No obstante, prende su propio nido y a sí misma y de sus cenizas vuelve a renacer de nuevo. De esta manera sucede que, aunque siempre muere, siempre vive. 